\title{
Study of the effect of dietary counselling on the improvement of end-stage renal disease patients
}

\author{
I.S. Hegazy, ${ }^{1}$ H.A. El Raghy, ${ }^{1}$ S.B. Abdel-Aziz' and E.M. Elhabashi'
}

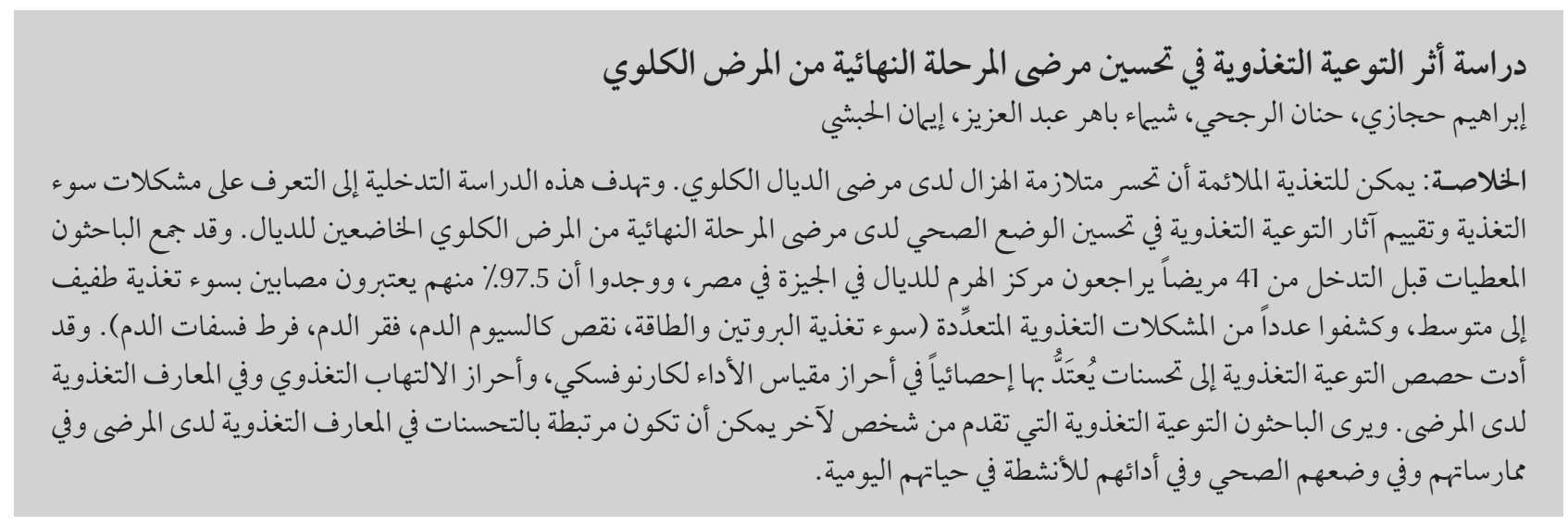

ABSTRACT Proper nutrition may help to reverse the wasting syndrome in dialysis patients with kidney disease on dialysis. This intervention study aimed to identify malnutrition problems and assess the effect of dietary counselling on improvement of health status of end-stage renal disease patients subjected to haemodialysis. Pre-intervention data were collected from 41 patients attending El Haram dialysis centre, Giza, Egypt; 97.5\% of the patients were considered mildly to moderately malnourished and multiple malnutrition problems were detected (protein-energy malnutrition, hypocalcaemia, and anaemia and hyperphosphataemia). Nutritional counselling sessions resulted in statistically significant post-intervention improvements in Karnofsky performance scale scores, malnutrition inflammation scores and nutritional knowledge of the patients. Providing one-to-one nutrition counselling could be linked to improvements in the patients' nutritional knowledge and practices and to their health status and performance in activities of daily life.

Étude de l'effet des conseils diététiques sur l'amélioration de l'état des patients atteints d'une maladie rénale en phase terminale

RÉSUMÉ Une alimentation adaptée peut contribuer à lutter contre le syndrome cachectique chez les patients atteints d'une maladie rénale sous dialyse. La présente étude d'intervention visait à identifier les problèmes de malnutrition et à évaluer l'effet de conseils diététiques sur l'amélioration de l'état de santé des patients atteints d'une maladie rénale en phase terminale soumis à une hémodialyse. Les données avant l'intervention ont été recueillies auprès de 41 patients fréquentant le centre de dialyse El Haram à Giza (Égypte) ; 97,5\% des patients étaient légèrement à modérément malnutris, et des problèmes de malnutrition multiples ont été détectés (malnutrition protéïno-énergétique, hypocalcémie, anémie et hyperphosphatémie). Des sessions de conseils nutritionnels ont entraîné des améliorations statistiquement significatives dans les scores sur l'indice de performance de Karnofsky, les scores de l'inflammation et de malnutrition et les connaissances diététiques des patients après l'intervention. L'offre de conseils nutritionnels en entretien individuel a entraîné des améliorations dans les connaissances diététiques des patients et dans leurs pratiques en la matière. Elle a également permis d'améliorer leur état de santé et leur performance dans leurs activités quotidiennes.

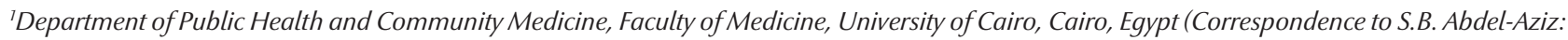
shaimaabaher@yahoo.com).

Received: 11/03/11; accepted: 11/01/12 


\section{Introduction}

Chronic kidney disease is a major public health problem and its incidence and prevalence are increasing [1]. Worldwide, the reported annual incidence of end-stage renal disease (ESRD) ranges between 34 and 200 per million population [2] and there is an even higher number of patients in the earlier stages of chronic kidney disease, facing adverse outcomes such as kidney failure, cardiovascular disease and premature death [3-5].

Malnutrition is common in patients with ESRD. Various studies have shown signs of malnutrition in $23 \%-76 \%$ of patients on haemodialysis and $18 \%-50 \%$ on peritoneal dialysis. Such variations in the prevalence of malnutrition may be related to factors such as age, comorbid conditions and quality of dialysis therapy. The aetiology of malnutrition in ESRD is complex and may include poor food intake because of anorexia, nausea and vomiting due to uraemia, hormonal derangements, acidosis and increased energy expenditure $[6,7]$. Proper nutrition may help to reverse the wasting syndrome. Several international studies have suggested a strong association between nutrition and clinical outcome in haemodialysis patients $[8,9]$.

Nutritional education and counselling for patients with renal disease plays a major role in the preservation of renal function and the overall wellbeing of the renal patient. In preparation for renal replacement therapy, a consultation with the renal nutritionist to establish a diet consistent with the existing diagnosis may increase the likelihood of reducing cardiovascular risk factors, preventing malnutrition and anaemia, and slowing the progression of renal disease, all of which can contribute to positive patient outcomes [10]. Innovations in nutrition education to patients have included a variety of approaches to deliver the message: one-to-one counselling, group counselling, involvement in patient and family support groups, cookery classes, recipes with food samples, posters, videos, quizzes, competitions, newsletters and report cards. Nutrition tips are another effective way of providing a practical nutrition education message in a simple format, where patients can make one small change at a time in their food choices [11].

In 2003 in Egypt the incidence of EDRD was reported as 74 per million [4]. The current study aimed to identify malnutrition problems and assess the effect of dietary counselling on the improvement of health status of ESRD patients attending a dialysis centre in Egypt.

\section{Methods}

\section{Study setting and sample}

This was an intervention study carried out at El Haram dialysis centre, Giza, Egypt from 1 October 2008 to 31 March 2009. This dialysis centre has 12 haemodialysis machines unit and is managed by the Egyptian health insurance organization.

Eligible patients were all those undergoing regular haemodialysis at the centre, i.e. 3 times per week every alternate day with Friday off. At the time of the study there were 83 patients ( 56 males and 27 females). A sex-stratified random sample of about $50 \%$ of the total dialysis patients were selected and enrolled in phase 1 of the study: 41 patients (28 males and 13 females). The number of patients in the follow-up (phase 3) was reduced to 37 as $2 \mathrm{pa}$ tients died, 1 patent was in the intensive care unit and 1 was hospitalized for an eye operation.

The study was conducted after explaining the steps of the study and its objectives to the participants. Only those who agreed were included. Verbal consent was obtained from all the participants in the study.

\section{Study design}

The study was conducted in several phases:

- Phase 1 (pre-intervention): collection of baseline data through interviews and laboratory reports with patients attending the centre to assess their nutritional status.

- Phase 2 (intervention): nutritional counselling that involved communicating evidence-based practices to all the patients in one-to-one sessions delivered by the researcher. Renal failure dietary guidelines tables [12] were used to show different types, quantities of food categories and the importance of each food group for health. Eating habits that needed to be emphasized or changed were discussed, e.g. the importance of fluid restriction and restricted salt intake. Each session involved the use of a laptop computer showing slides with nutritional messages and animations showing the importance of fluid restriction. Printed materials with all messages were distributed at the end of each session to the patients, the doctors and the nurses. Printouts of important general information about diabetes were also distributed to diabetic ESRD patients to emphasize the importance of controlling diabetes.

- Phase 3 (post-intervention): a reassessment of the impact of nutritional counselling through follow-up interviews and the latest laboratory reports. This was conducted about 1 month later to allow sufficient time for assimilation and implementation of the taught messages.

\section{Data collection}

We met each patient 5 times over the course of the research, in order to conduct the pre- and post-intervention interviews and the nutritional counselling sessions. These were scheduled around the various dialysis shifts/groups.

\section{Interview data}

Baseline data were collected from the study sample using an interview 
questionnaire containing the following sections: general information, sociodemographic data, medical history, dietary pattern, nutritional status and health-related quality of life/functional impairment.

Functional impairment and quality of life was assessed using the Karnofsky performance status scale index [13] which rates patients' ability to carry on normal activity and work and their need for assistance with personal needs or for institutional or hospital care on a scale from 0 (dead) to 100 (normal no complaints; no evidence of disease). Patients' functional impairment scores were classified as follows: normal (80$100)$, mild (50-80), moderate (25-50) and severe $(\leq 25)$.

Patients' nutritional status was assessed based on history taking using the malnutrition inflammation score (MIS) [14]. This has 10 items in 2 sections: patient's related medical history [change in end dialysis dry weight (in past 3-6 months); dietary intake; gastrointestinal symptoms; nutritionally related functional impairment; comorbidity (including number of years on dialysis)] and physical examination [decreased fat stores or loss of subcutaneous fat; signs of muscle wasting; body mass index (BMI); serum albumin, serum total iron binding capacity or serum transferring]. Only 6 out of the 10 MIS components were used and total scores were classified as follows: normal $(\leq 1)$, mild (1-8) and moderate $(8-18)$. Weight and height were measured and BMI was calculated and classified according to World Health Organization cut-offs: underweight $(<$ $\left.18.5 \mathrm{~kg} / \mathrm{m}^{2}\right)$, normal $(18.5-24.9 \mathrm{~kg} /$ $\left.\mathrm{m}^{2}\right)$, overweight $\left(25.0-29.9 \mathrm{~kg} / \mathrm{m}^{2}\right)$, obese $\left(30.0-39.9 \mathrm{~kg} / \mathrm{m}^{2}\right)$ and morbid obese $\left(>40 \mathrm{~kg} / \mathrm{m}^{2}\right)$.

Dietary patterns of patients were estimated from patients' 24-hour recall using to the National Nutrition Institute methods [15]. Patients' were classified according to their daily nutrition requirements into 5 classes: unsafe $(\leq 50 \%)$, needs improvement $(50 \%-$ $75 \%)$, acceptable (75\%-100\%), normal $(100 \%-120 \%)$ and unacceptable $(\geq$ $120 \%)$.

\section{Biochemical data}

For the biochemical analysis blood samples were taken at the pre- and postintervention interviews for estimation of serum urea, serum creatinine, serum calcium, serum phosphorus and red cell haemoglobin levels.

\section{Statistical analysis}

Data were computerized and analysed using SPSS, version 16 software. Simple statistics such as frequencies, percentages, arithmetic means and standard deviation (SD) were used. Comparison between pre- and postassessment values was done using the McNemar test for qualitative variables and paired Student $t$-test for quantitative variables.

\section{Results}

\section{Background characteristics}

This study sample included 41 patients ( 13 female and 28 male), i.e. a male to female ratio of 2.1. The age of the study group ranged from $27-75$ years, mean age was 51.7 (SD 12.6) years, $43.9 \%$ of the study sample were illiterate and $73.1 \%$ were not working, $92.7 \%$ were married. Just over half the sample were smokers (53.7\%).

A great majority of ESRD patients (90.2\%) had hypertension, $17.1 \%$ were diagnosed with diabetes and $9.8 \%$ with cardiovascular disease. Nearly half of them had hepatitis $\mathrm{C}$ virus (43.9\%) and $4.9 \%$ had bilharziasis. Almost all the patients were suffering from bleeding problems and impaired wound healing and 30\%-60\% reported symptoms such as fatigue and malaise, headache, weight loss, muscle wasting, frequent infections and bone problems.

The duration of dialysis was more than 2 years for $70 \%$ of them. There was a first-degree family history of ESRD in $9.8 \%$ of the patients.

\section{Pre- and post-intervention comparisons}

\section{Nutritional assessment}

The nutritional background and dietary profile showed that before the intervention only $29.3 \%$ of patients on dialysis received nutritional sessions that were conducted by doctors on an occasional basis. The majority of the sample (90.2\%) knew nothing about nutritional assessment methods. The most common reasons for not following a dietary regime were appetite issues or ignorance, followed by social issues and, in a few cases, financial issues.

According to the patients' 24-hour recall of dietary habits (National Nutrition Institute classification) one-third of them (34.1\%) needed improvement in their protein intake and were suffering from an unsafe sodium intake, $51.2 \%$ had an unacceptable intake of potassium and were suffering from an unsafe phosphorus intake, while $20.0 \%$ were suffering from unsafe calorie intake that needed improvement. It was noted that $90.2 \%$ of the patients had an unaccepted level of fluid intake. According to the National Nutrition Institute classification only $48.8 \%$ of patients were practising healthy eating habits before the intervention This increased from to $78.4 \%$ post-intervention $(P<0.002)$ (Table 1).

\section{Nutritional knowledge}

Table 1 shows a comparison of patients' nutritional knowledge pre- and postintervention. There were improvements in all items of knowledge post-intervention, although these were not statistically significant for the definitions of malnutrition and how malnutrition can affect ESRD patients' health status. "Don't know" responses to the item on knowledge about the effect of malnutrition on health decreased dramatically from $82.9 \%$ to only $5.4 \%$ after the intervention and the item concerning knowing 


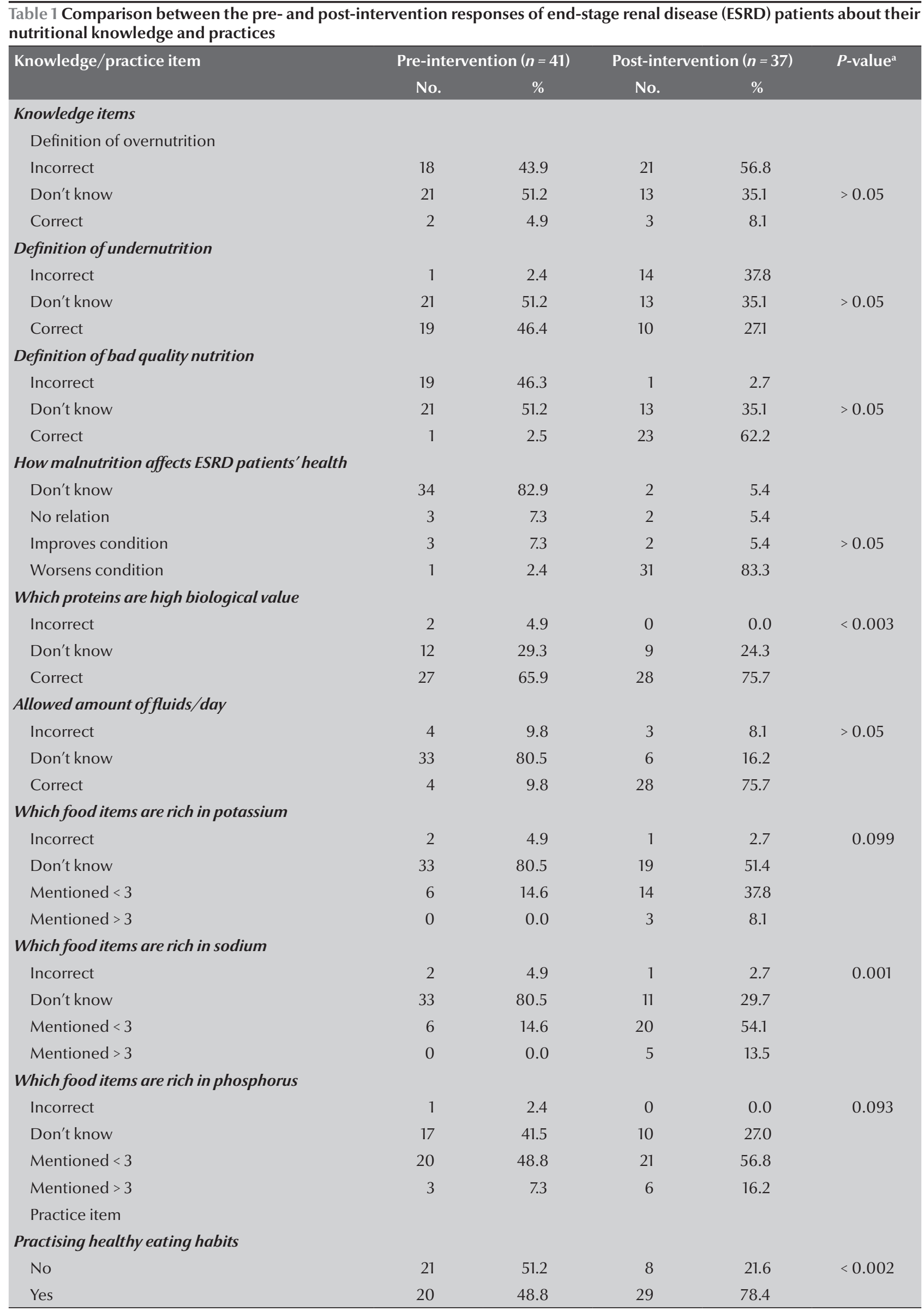

${ }^{a} \mathrm{McNemar}$ test. 
how far this relationship can worsen the condition increased from $2.4 \%$ to $83.3 \%$ (not significant, McNemar test). There was a significant improvement between the pre- and post-intervention phases in the proportion of patients who knew which proteins were of high biological value $(75.5 \%$ versus $65.9 \%)(P<0.003)$ (Table 1). The percentage who knew the right allowed amount of fluids that should be taken every day also increased from $9.8 \%$ to $75.7 \%$ (not significant, McNemar test). Patients' knowledge about food items rich in certain minerals increased post-intervention. For example, the proportion who mentioned more than 3 foods rich in potassium increased from $0 \%$ to $8.1 \%$ and for foods rich in phosphorus from $7.3 \%$ to $16.2 \%$ $(P>0.05)$, while there was a significant increase in those who mentioned more than 3 foods rich in sodium from $0 \%$ to $13.5 \%(P<0.001)$.

\section{Biochemical parameters}

Comparison of patients' biochemical parameters showed statistically significant decreases in the mean values of creatinine, urea and calcium before and after the intervention (paired Student t-test) (Table 2). Phosphorus levels decreased and haemoglobin levels increased but these differences were not significant.

\section{Karnofsky performance scale}

The proportion of patients within the normal band of the Karnofsky functional impairment scale was only $22.0 \%$ before the intervention but this rose significantly to $78.4 \%$ post-intervention, while those in the mild band decreased from $73.2 \%$ pre- to $18.9 \%$ post-intervention. The mean Karnofsky scale score (range 0-100) for 41 patients before the intervention was 70.5 (SD 8.5) and for the 37 patients after the counselling was 78.1 (SD 9.9).

\section{Malnutrition inflammation score}

Before the intervention the great majority of patients $97.5 \%$ were considered to be mild to moderate malnourished and only $2.5 \%$ were well nourished using the MIS score (Table 3). After the intervention, the proportion of patients within the mild band remained almost the same (90.2\% and 91.9\%) while the percentage who were well nourished increased to $8.1 \%$ post-intervention (Table 3). The proportion in the moderate band decreased from $7.3 \%$ preintervention to $0 \%$ post-intervention. The mean MIS score (range 0-18) before the intervention was 4.6 (SD 1.9) and after the counselling was 3.1 $(\mathrm{SD} 1.7)(P<0.001)$.

\section{Discussion}

Malnutrition is a major comorbid condition in ESRD, with a prevalence of $40 \%-50 \%$ in patients with ESRD $[10,16,17]$. Malnutrition compromises the prognosis in ESRD, and nutritional status is therefore an important predictor of outcomes for patients on maintenance haemodialysis. Assessment of nutritional status needs a systematic nutritional evaluation based on anthropometric, laboratory and clinical parameters from which a malnutrition score can be calculated [18]. Medical nutrition therapy and nutrition education and counselling have been shown to be essential components of effective management of ESRD. Early nutritional intervention is thought to play a major role in the preservation of renal function and the overall well-being in the renal patient $[10,19]$. Based on this evidence, the current research was carried out with the aim of revealing the effects of dietary counselling on improving the health status of dialysis patients.

Half of the patients at our dialysis centre were enrolled in this study and the mean age of patients was 51.7 (SD 12.6) years. Previous national studies in Egypt found a mean age of 43.0 (SD 17.7) years in 1987 by Mohammed [20] and a mean of 45.6 (SD 14.2) years in 1999 [21]. The higher mean age of ESRD patients in our study in Egypt reflects the universal trend towards dialysis patients living longer due to improving health care [21].

Our study showed that $90.2 \%$ of the sample were suffering from hypertension and $17.1 \%$ from diabetes mellitus, diseases that are considered the $2 \mathrm{ma}-$ jor risk factors of renal failure. Webster Gandy et al. and Moriyama also reported that the most common risk factors for kidney failure were diabetes and hypertension, together accounting for almost $69 \%$ of new cases [5]. In Egypt hypertension was reported to be responsible for $28 \%$ of cases of ESRD in 1996 and for 22\% in 2001 [21] and in Yemen for $30 \%$ of cases [17]. The

\begin{tabular}{|c|c|c|c|c|c|}
\hline \multirow[t]{2}{*}{ Parameter } & \multicolumn{2}{|c|}{ Pre-intervention $(n=41)$} & \multicolumn{2}{|c|}{ Post-intervention $(n=37)$} & \multirow[t]{2}{*}{$P$-value ${ }^{\text {a }}$} \\
\hline & Mean & SD & Mean & SD & \\
\hline Creatinine (mg/dL) & 9.6 & 3.2 & 8.3 & 2.8 & $<0.001$ \\
\hline Urea $(\mathrm{mg} / \mathrm{dL})$ & 141.6 & 39.8 & 128.0 & 38.3 & $<0.031$ \\
\hline Calcium (mg/dL) & 7.5 & 1.1 & 6.8 & 0.9 & $<0.002$ \\
\hline Phosphorus (mg/dL) & 4.8 & 1.6 & 4.4 & 1.5 & $>0.05$ \\
\hline Haemoglobin $(\mathrm{g} / \mathrm{dL})$ & 10.7 & 2.3 & 11.4 & 2.1 & $>0.05$ \\
\hline
\end{tabular}

apaired Student t-test.

$S D=$ standard deviation . 


\begin{tabular}{|c|c|c|c|c|c|}
\hline \multirow[t]{2}{*}{ Scale/scores } & \multicolumn{2}{|c|}{ Pre-intervention $(n=41)$} & \multicolumn{2}{|c|}{ Post-intervention $(n=37)$} & \multirow[t]{2}{*}{$P$-value } \\
\hline & No. & $\%$ & No. & $\%$ & \\
\hline \multicolumn{6}{|c|}{ Karnofsky scale score ${ }^{a, b}$} \\
\hline Normal (80-100) & 9 & 22.0 & 29 & 78.4 & $<0.001$ \\
\hline Mild (50-80) & 30 & 73.2 & 7 & 18.9 & \\
\hline Moderate (25-50) & 2 & 4.9 & 1 & 2.7 & \\
\hline Mean (SD) & \multicolumn{2}{|c|}{$70.5(8.5)$} & \multicolumn{2}{|c|}{$78.1(9.9)$} & \\
\hline \multicolumn{6}{|c|}{ Malnutrition inflammation score ${ }^{a, c}$} \\
\hline Normal $(\leq 1)$ & 1 & 2.5 & 3 & 8.1 & \\
\hline Mild (1-8) & 37 & 90.2 & 34 & 91.9 & $<0.001$ \\
\hline Moderate (8-18) & 3 & 7.3 & 0 & 0.0 & \\
\hline Mean (SD) & \multicolumn{2}{|c|}{$4.6(1.9)$} & \multicolumn{2}{|c|}{$3.1(1.7)$} & \\
\hline
\end{tabular}

${ }^{a}$ No cases were found within severe band; ${ }^{b}$ Range 0-100; ${ }^{c}$ Modified scale, range 0-18. ${ }^{d}$ Student $t$-test.

$S D=$ standard deviation .

high incidence of hypertension in our study is consistent with reports from the United States, Japan and Germany and other European countries [22].

Poor nutritional status of haemodialysis patients is the result of several interrelated factors. Apart from the catabolic effect of dialysis, nutrient loss in dialysis and uraemic toxicity, several comorbid conditions may also contribute to malnutrition, including chronic infection and superimposed diseases that result in anorexia and inadequate food intake [23]. It was also observed from the present study and that of Afshar et al. [9] that many patients were suffering from symptoms related to malnutrition such as fatigue and malaise, headache, weight loss, muscle wasting, frequent infections, impaired wound healing and bone troubles. This finding can be compared with Basaleem et al.'s study, where all the 50 study patients were suffering of bone pains and arthralgia [17]. Moe et al. also reported that renal osteodystrophy was common in ESRD patients and related to disturbance in bone and mineral metabolism [24].

In the present study, $97.5 \%$ of patients were considered to be mild to moderate malnourished at baseline and only $2.5 \%$ were well-nourished according to the MIS score. A study in Yemen reported that out of 50 patients $70 \%$ of them had moderate malnutrition and $20 \%$ were severely malnourished [17]. Other international data showed that up to $76 \%$ of maintenance haemodialysis patients were malnourished [6]. The higher percentage of malnutrition in our study may be explained by the fact that patients showed a very low level of nutritional knowledge at baseline. After nutritional counselling, however, a statistically significant improvement was observed as regard patients' knowledge about nutrition and how malnutrition can affect their general health. Patients' knowledge increased for the items about the allowed amount of fluids per day and their compliance with it, about proteins of high biological value, about foods rich in sodium, phosphorus and potassium and the risk of malnutrition. It is likely that this can be attributed to the intervention in our study. Our results are similar to the programme implemented in Canada by Berg et al., who found improvements in patients' nutritional knowledge and their salt and fluid intakes after an educational programme [11]. They used an incentive method in the form of a lotto draw for patients who did not exceed their recommended interdialytic weight and, as in our study, they distributed nutritional tips aiming to improve patients' nutritional status.

Analysis of the data about patients' nutritional practices also showed an improvement in different aspects of their diet after the intervention. Before the intervention $51.2 \%$ of the sample were not practising healthy eating habits, a figure that is similar to Basaleem et al.'s study in which $58 \%$ of the sample were not following dietary instructions [17]. This may be explained by the finding that only $29.3 \%$ of our patients reported ever receiving nutritional education sessions before the intervention and these were not on a regular basis. The most common reasons for not following a dietary regimen were due to lack of appetite or to ignorance. So different tips and ideas were given in the counselling to help patients with the social, appetite and financial issues, each according to his personal problem, while for those with poor knowledge counselling acted to raise their knowledge level.

Abnormal levels of biochemical parameters are usually encountered in patients with ESRD, some of which (e.g. high blood urea and creatinine) are predictors of mortality. In the present 
study a statistically significant decline in creatinine, urea, calcium and phosphorus levels were detected comparing before and after the intervention and this is similar to what was reported by Basaleem et al. [17].

To summarize, providing one-toone nutrition counselling in our study was followed by an improvement in patients' nutritional knowledge and practice and improvement of their compliance with dietary guidelines for the dialysis patient, which in turn could be linked to significantly improvement in their health status and performance in activities of daily life.
Acknowledgements

We thank all health care providers in El Harm Centre for Dialysis for their cooperation and all staff colleges in public health department of the medical college, University of Cairo, Egypt for their continuous support.

\section{References}

1. Levey AS et al. Definition and classification of chronic kidney disease: a position statement from kidney disease: improving global outcomes (KDIGO). Kidney International, 2005, 67:2089-2100.

2. Eknoyan $\mathrm{G}$ et al. The burden of kidney disease, improving global outcomes. Kidney International, 2004, 66:1310-131.

3. Barsoum RS. End-stage renal disease In North Africa, Cairo, Egypt. Kidney International, 2003, 63:S111-S114.

4. Collins AJ et al. Excerpts from the United States Renal Data System 2006 annual data report. American Journal of Kidney Disease, 2007, 49(1 Suppl. 1):A6-7, S1-296.

5. Moriyama T. [Causes and characteristics of chronic kidney disease.] Japanese Journal of Clinical Medicine, 2008, 66:16641670 [in Japanese].

6. Stenvinkel $\mathrm{P}$ et al. Are there two types of malnutrition in chronic renal failure? Evidence for relationships between malnutrition, inflammation and atherosclerosis (MIA syndrome). Nephrology, Dialysis, Transplantation, 2000, 15:953-960.

7. Alpers DH et al., eds. Manual of nutritional therapeutics, 4th ed. Philadelphia, Lippincott, Williams and Wilkins, 2002.

8. Qureshi AR et al. Inflammation, malnutrition, and cardiac disease as predictors of mortality in hemodialysis patients. Journal of the American Society of Nephrology, 2002, 13(Suppl. 1):S28-S36.

9. Afshar R, Sanavi S, Izadi-Khah A. Assessment of nutritional status in patients undergoing maintenance hemodialysis: a single-center study from Iran. Saudi Journal of Kidney Diseases and Transplantation, 2007, 18:397-404.

10. Moore $\mathrm{H}$ et al. National Kidney Foundation Council on Renal Nutrition survey: past-present clinical practices and future strategic planning. Journal of Renal Nutrition, 2003, 13:233-240.

11. Berg J et al. Incentive program to control interdialytic weight gains. Journal of Renal Nutrition, 2004, 14(1):52-59.

12. Webster-Gandy J, Madden A, Holdsworth M. Oxford handbook of nutrition and dietetics, 2nd ed. Oxford, Oxford University Press, 2008.

13. Karnofsky DA, Burchenal JH. The clinical evaluation of chemotherapeutic agents in cancer. In: MacLeod CM, ed. Evaluation of chemotherapeutic agents. Columbia, Columbia University Press, 1949:196.
14. Kalantar-Zadeh $\mathrm{K}$ et al. A malnutrition inflammation score is correlated with morbidity and mortality in maintenance hemodialysis patients. American Journal of Kidney Disease, 2001, 38:1251-1263.

15. Dietary guidelines for Americans. Unite States Department of Health and Human Services [website] (http://health.gov/ dietaryguidelines/, accessed 5 November 2012).

16. Cano N. [Malnutrition and chronic renal failure]. Denutrition et insuffisance renale chronique. Annales de Medecine Interne, 2000, 151:563-574.

17. Basaleem $\mathrm{HO}$ et al. Assessment of the nutritional status of endstage renal disease patients on maintenance hemodialysis. Saudi Journal of Kidney Diseases and Transplantation, 2004, 15:455-462.

18. Schulman G. Nutrition in daily hemodialysis. American Journal of Kidney Diseases, 2003, 41(Suppl. 1):S112-S115.

19. Burrowes JD. Incorporating ethnic and cultural food preferences in the renal diet. Advances in Renal Replacement Therapy, 2004, 11:97-104.

20. Afifi A, Karim MA. Renal replacement therapy in Egypt: first annual report of the Egyptian Society of Nephrology, 1996. Eastern Mediterranean Health Journal, 1999, 5(5):1023-1029.

21. Mohammed N. Tuberculosis in chronic renal failure patients under dialysis treatment [thesis]. Cairo, Egypt, Ain Shams University, 1987.

22. Mallick NP, Jones E, Selwood N. Annual report on management of renal failure in Europe XXVII, 1996. XXXIVth Congress of the European Renal Association-European Dialysis and Transplantation Association. Geneva, 21-24 September 1997. American Journal of Kidney Diseases, 1997, 30(Suppl. 1):S1-213.

23. Stenvinkel $\mathrm{P}$ et al. A Comparative analysis of nutritional parameters as predictor of outcome in male and female ESRD patients. Sweden, Nephrology, Dialysis, Transplantation, 2002, 17:1266-1274.

24. Moe $\mathrm{S}$ et al. Definition, evaluation, and classification of renal osteodystrophy: a position statement from Kidney Disease: Improving Global Outcomes (KDIGO). Kidney International, 2006, 69:1945-1953. 